\title{
TTR
}

Traduction, terminologie, rédaction

\section{Relire l'histoire de la traduction littéraire au Canada : d'une tradition de traduction à des amorces de traditions imprévisibles}

\section{Gillian Lane-Mercier}

Volume 31, numéro 2, 2e semestre 2018

Minorité, migration et rencontres interculturelles : du binarisme à la complexité

Minority and Migrant Intercultural Encounters: From Binarisms to

Complexity

URI : https://id.erudit.org/iderudit/1065567ar

DOI : https://doi.org/10.7202/1065567ar

Aller au sommaire du numéro

Éditeur(s)

Association canadienne de traductologie

ISSN

0835-8443 (imprimé)

1708-2188 (numérique)

Découvrir la revue

Citer cet article

Lane-Mercier, G. (2018). Relire l'histoire de la traduction littéraire au Canada : d'une tradition de traduction à des amorces de traditions imprévisibles. TTR, 31(2), 17-42. https://doi.org/10.7202/1065567ar
Résumé de l'article

À partir de la définition de deux collaborations à visée interculturelle prototypiques des sociétés démocratiques multilingues et multiculturelles contemporaines, l'une fondée sur le consensus, l'autre sur le compromis, cet article propose une analyse des enjeux soulevés par cette double conception de la participation citoyenne. Plus spécifiquement, le premier objectif consiste à examiner le rôle de la traduction littéraire dès lors susceptible de servir deux projets de société qui prônent, respectivement, l'assimilation culturelle, fondée sur la banalisation des rapports de domination au nom de l'égalité, et l'intégration, fondée sur la nécessité de donner droit de cité aux inégalités afin d'assurer la visibilité et la vitalité, sinon la survie des communautés minoritaires. Nous nous penchons à titre d'exemple sur le rôle de la traduction littéraire dans l'évolution des rapports difficiles entre la majorité francophone et la minorité anglophone du Québec depuis 1976, en particulier dans la revitalisation culturelle de celle-ci au cours des vingt dernières années. Aussi le deuxième objectif consiste-t-il à remettre en question une certaine lecture de l'histoire de ces rapports qui, postulant l'émergence d'une tradition de traduction où domine le consensuel, repose moins sur des faits que sur des perceptions, discours et idées reçues transformés en évidences. La relecture proposée fait ressortir les compromis réels que ces « évidences » escamotent, ainsi que le rôle de la traduction littéraire comme solution de compromis dans le processus de revitalisation en situation minoritaire. Pour ce faire, et en admettant que la traduction littéraire relève d'une dialectique du fait empirique et du construit discursif, nous recourons à la bibliométrie qui permet ainsi d'établir une articulation qui manque encore entre l'histoire de la traduction, axée sur les faits empiriques, et la traductologie, axée sur les discours.
Ce document est protégé par la loi sur le droit d'auteur. L’utilisation des services d’Érudit (y compris la reproduction) est assujettie à sa politique d'utilisation que vous pouvez consulter en ligne.

https://apropos.erudit.org/fr/usagers/politique-dutilisation/ 


\title{
Relire l'histoire de la traduction littéraire au Canada : d'une tradition de traduction à des amorces de traditions imprévisibles ${ }^{1}$
}

\section{Gillian Lane-Mercier}

Université McGill

\begin{abstract}
Résumé
À partir de la définition de deux collaborations à visée interculturelle prototypiques des sociétés démocratiques multilingues et multiculturelles contemporaines, l'une fondée sur le consensus, l'autre sur le compromis, cet article propose une analyse des enjeux soulevés par cette double conception de la participation citoyenne. Plus spécifiquement, le premier objectif consiste à examiner le rôle de la traduction littéraire dès lors susceptible de servir deux projets de société qui prônent, respectivement, l'assimilation culturelle, fondée sur la banalisation des rapports de domination au nom de l'égalité, et l'intégration, fondée sur la nécessité de donner droit de cité aux inégalités afin d'assurer la visibilité et la vitalité, sinon la survie des communautés minoritaires. Nous nous penchons à titre d'exemple sur le rôle de la traduction littéraire dans l'évolution des rapports difficiles entre la majorité francophone et la minorité anglophone du Québec depuis 1976, en particulier dans la revitalisation culturelle de celle-ci au cours des vingt dernières années. Aussi le deuxième objectif consiste-t-il à remettre en question une certaine lecture de l'histoire de ces rapports qui, postulant l'émergence d'une tradition de traduction où domine le consensuel, repose moins sur des faits que sur des perceptions, discours et idées reçues transformés en évidences. La relecture proposée fait ressortir les compromis réels que ces «évidences» escamotent, ainsi que le rôle de la traduction littéraire comme solution de compromis
\end{abstract}

1. Cet article relève d'un projet en cours et s'inscrit dans une série d'articles publiés depuis 2014. Plusieurs éléments ont été abordés plus en détail ailleurs, dont la notion de tradition de traduction (Lane-Mercier, 2014a), les problèmes méthodologiques (Lane-Mercier, 2014b), la notion de collaboration (LaneMercier, 2017) et les politiques de traduction officielles au Canada (LaneMercier, 2018a). D'autres éléments ont été repris en vue d'une mise à jour des données empiriques et de leur interprétation ou encore de fournir un contexte à la démonstration (Lane-Mercier, 2018b). 
dans le processus de revitalisation en situation minoritaire. Pour ce faire, et en admettant que la traduction littéraire relève d'une dialectique du fait empirique et du construit discursif, nous recourons à la bibliométrie qui permet ainsi d'établir une articulation qui manque encore entre l'histoire de la traduction, axée sur les faits empiriques, et la traductologie, axée sur les discours.

Mots-clés : traductologie, histoire de la traduction, traduction littéraire et revitalisation culturelle, traduction littéraire en situation minoritaire, traduction littéraire au Canada

\section{Abstract}

This article analyses the issues raised by the definition of two prototypical intercultural collaborations, one grounded in consensus and the other in compromise, which have fostered competing conceptions of participatory citizenship in contemporary multilingual and multicultural democratic societies. Specifically, the first objective is to examine the role of literary translation, henceforth called upon to serve two social contracts that advocate, respectively, cultural assimilation based on the trivialization of power relations in the name of equality, and cultural integration based on the importance of acknowledging inequalities to ensure the visibility, vitality and, at times, survival of minority communities. We take as an example the role of literary translation in the evolution of the difficult relations between Québec's Francophone majority and Anglophone minority since 1976, more particularly in the cultural revitalization the latter has experienced over the past twenty-odd years. Thus, the second objective is to call into question a well-oiled reading of the history of these power relations that, by positing the emergence of a tradition of translation predicated on consensus, is founded less on fact than on perceptions, ambient discourses and received ideas construed as truths. The rereading proposed here exposes the real compromises which these "truths" erase, as well as the role of literary translation as concession in the cultural revitalization of the Anglophone communities. Bibliometrics provides us with a heuristic tool to attain these objectives, with the additional benefit of creating a much needed link between the history of translation, which focuses on empirical facts, and translation studies, which focuses primarily on discursive constructs.

Keywords: translation studies, history of translation, literary translation and cultural revitalization, literary translation in minority situations, literary translation in Canada

\section{Revitalisation interculturelle et traduction littéraire : consensus ou compromis?}

Si la vitalité d'une communauté linguistique minoritaire relève de prime abord de facteurs démographiques, institutionnels et législatifs (v. Fraser, 2008; Jedwab, 2008), on ne saurait sous-estimer l'importance de facteurs d'ordre plus spécifiquement culturel. Certains 
signes de vitalité culturelle se manifestent sous forme d'initiatives visant à consolider l'infrastructure (par exemple les maisons d'édition, les théâtres) nécessaire à la reconnaissance des artistes et des artisans minoritaires par les membres de leur propre communauté. D'autres signes de vitalité culturelle se manifestent sous forme de collaborations entre la culture majoritaire et la culture minoritaire, en vue d'assurer à cette dernière une visibilité, voire un sentiment d'appartenance collective à la fois distinct de et corrélé à la culture dominante. Distinguons d'entrée de jeu deux collaborations prototypiques.

D'une part, les collaborations à visée interculturelle peuvent protéger contre l'assimilation, à condition de voir dans les rapports de domination qui les sous-tendent un vecteur dynamique et positif d'intégration participative. Celle-ci est alors axée sur le compromis, défini comme «un processus de concessions réciproques» (Weinstock, 2011, p. 235; italique dans l'original) dont peuvent tirer avantage les cultures minoritaires (v. Cardinal, 2001), à plus forte raison lorsque les communautés en contact ne partagent pas la même langue. Dans cette optique, posons d'emblée que la traduction littéraire représente une activité collaborative interculturelle destinée à assurer la reconnaissance mutuelle, à (re)négocier les rapports de force et à favoriser l'auto-détermination culturelle des minorités. La traduction permet ainsi de respecter la notion de participation citoyenne à la base des sociétés démocratiques multilingues en général et des sociétés fédérales pluralistes en particulier (v. Meylaerts, 2009, 2011).

D'autre part, tout projet collaboratif porte en lui les germes de son échec, symbolique ou réel, à partir du moment où il se donne le consensus pour idéal régulateur. Misant sur le dépassement des tensions, déséquilibres et antagonismes au nom de l'égalité, des droits individuels et d'un avenir commun,

la quête du consensus révèle une trivialisation du pluralisme [...] [aux] conséquences contre-productives. En effet, si les participants dans une délibération démocratique considèrent l'atteinte du consensus comme le seuil du succès, ils auront tendance à mépriser et à dévaloriser les résultats plus limités qui se situeraient en deçà de ce seuil [...]. (Weinstock, 2001, p. 233-234)

D'où le caractère intéressé (assimilateur) des collaborations interculturelles régies par le consensus. Car chercher à dépasser différends et différences revient à minimiser la dynamique positive des rapports de pouvoir, garante de la possibilité même de la revitalisation cultu- 
relle et linguistique d'une communauté minoritaire, au profit de la force du nombre et de l'idéologie dominante. Comme le souligne Linda Cardinal (2001, p. 290), le principe de l'égalité des langues empêche les groupes d'entrer en relation².

En trivialisant la diversité effective pourtant au cœur de bon nombre de discours officiels actuels, les collaborations interculturelles qui visent le consensus privilégient l'unité, la non-différentiation et la parité, au détriment des revendications culturelles (et politiques) des communautés minoritaires. À la limite, la notion même de collaboration interculturelle s'avère inopérante. Posons donc, dans un deuxième temps et en reprenant à quelques mots près la formulation ci-dessus, que la traduction littéraire peut représenter une activité collaborative interculturelle destinée à banaliser les rapports de domination, sinon à les occulter. La traduction permet ainsi de respecter la notion de participation citoyenne à la base des sociétés démocratiques multilingues en général et des sociétés fédérales pluralistes en particulier.

\section{Traductologie et histoire de la traduction littéraire}

Nous voilà face à un paradoxe d'ordre traductologique. En contexte plurilingue et pluriculturel, la traduction littéraire incarne la valeur démocratique de participation citoyenne tantôt par le biais du compromis, fondé sur les droits collectifs (territoriaux) et la possibilité de (re)vitalisation culturelle des communautés minoritaires, tantôt par le biais du consensus, fondé sur les droits individuels (universels) et la minimisation de la (re)vitalisation culturelle. Autrement dit, la traduction est susceptible de servir deux projets de société collaboratifs prototypiques qui prônent, respectivement, l'intégration et l'assimilation (v. Cronin, 2006; Vázquez, 2011) .

Au vu de ce paradoxe, il est indispensable de se doter d'outils méthodologiques aptes à appréhender, dans une perspective traductologique et historique, les enjeux soulevés par cette double conception

2. «[L]es normes et les lois, se drapant dans les oripeaux de la neutralité, traînent avec elles la trace de la majorité même lorsqu'elles sont passées par l'épreuve de la délibération» (Maclure, 2001, p. 260).

3. Signalons un troisième idéal de régulation en régime démocratique : le recours à des stratégies non collaboratives, dont la résistance, la dissension ou le veto (v. Weinstock, 2001). Or, si la traduction peut être acte de résistance radical, elle contient encore un coefficient d'interculturalité. Seule la non-traduction relève sans ambages de la non-collaboration interculturelle. 
de la participation citoyenne, ainsi que les stratégies institutionnelles qui l'étayent. Aussi faut-il mettre au jour, dans le cadre de traductions consensuelles où prime le principe de l'égalité des langues-cultures, les déséquilibres et tensions qui continuent à se déployer en sourdine dans les angles morts d'une activité collaborative qui sert certains intérêts plutôt que d'autres et, dans le cadre de traductions où priment les principes de non-égalité et de différence, les modalités selon lesquelles la traduction se transforme en «a vehicle for cultural survival» (Baumgarten et Gruber, 2014, p. 29). Car les rapports de pouvoir en situation plurilingue minoritaire sont salutaires lorsque, réfractaires au consensus, ils alimentent les collaborations interculturelles en vue de donner droit de cité aux inégalités réelles et aux solutions de compromis qu'elles requièrent. Historiquement associés aux exclusions et autres effacements culturels plus ou moins définitifs, les rapports de domination contiennent aussi un potentiel positif de (re)vitalisation et d'auto-détermination que, en tant que minorité linguistique, il importe d'activer. À ce titre, la traduction littéraire soulève des questions particulièrement intéressantes dans les pays officiellement bi- ou multilingues pour autant qu'elle participe de la dynamique à la fois de jure (légiférée) et de facto (non légiférée) entre les communautés linguistiques en contact.

En schématisant à l'extrême, rappelons que les traductologues accordent la primauté au texte, qu'il soit source ou cible, conçu comme produit de multiples expériences, savoirs, normes et pratiques qu'il s'agit avant tout de conceptualiser. Pour leur part, les historiens de la traduction accordent la primauté aux processus diachroniques tributaires de faits empiriques (dates, lieux, textes, agents, institutions) qu'il s'agit avant tout de quantifier. L'un des défis actuels consiste à établir des articulations méthodologiques entre la traductologie, qui implique une approche qualitative (herméneutique, discursive), et l'histoire de la traduction, qui préconise une approche quantitative (empirique, statistique). Posons donc dans un troisième temps que la bibliométrie, méthode d'analyse statistique actuellement en plein essor dans le domaine de l'historiographie (v. D'hulst, 2014), fournit des instruments efficaces pour procéder à de telles articulations, créant ainsi une passerelle à double sens entre le mesurable (les faits identifiés par l'historien), l'interprétable (les analyses bibliométriques ou statistiques) et la conceptualisation (ce que le traductologue peut en dire). En d'autres termes, la bibliométrie offre des possibilités de "plausibilisation" empirique (historique) et théorique 
(traductologique) que nous proposons d'explorer dans les sections qui suivent en prenant pour exemple le Canada, officiellement bilingue depuis 1969 .

\section{La Bibliographie de livres canadiens traduits de l'anglais au français et du français à l'anglais}

Dans le contexte canadien-anglais, la traduction littéraire du français vers l'anglais a eu tendance à être perçue, jusqu'au milieu des années 1990, comme indice de la vitalité culturelle, souvent inquiétante sur le plan politique, de la société québécoise minoritaire et comme moyen de la faire connaître à la majorité anglophone 4 . Il s'agissait d'une véritable mission socio-politico-ethnographique que se sont conférée les traducteurs littéraires anglophones au nom de l'unité nationale. Inversement, dans le contexte québécois, la traduction littéraire de l'anglais vers le français a été perçue comme indice et moyen de dévitalisation (lire : colonisation) de la langue et de la culture des Québécois. D'où une attitude de méfiance et, chez beaucoup, une volonté de «nontraduction» (Brault, 1975), seule manière de protéger la culture de la minorité francophone contre l'assimilation par la majorité anglophone. Tout ceci est bien connu des historiens de la traduction littéraire au Canada (v. Simon, 1994); ajoutons simplement que ces attitudes, moins polarisées depuis une vingtaine d'années, ont été longtemps symptomatiques des rapports de force tendus depuis les années 1960 entre le Québec et le Canada anglais.

Parue en 1975, rééditée en 1977 au plus fort des tensions suscitées par le nationalisme québécois, la Bibliographie de livres canadiens traduits de l'anglais au français et du français à l'anglais établie par le comparatiste et traducteur littéraire anglophone Philip Stratford confirme, chiffres à l'appui, l'existence de ces attitudes. Non seulement deux fois plus d'œuvres avaient été traduites du français vers l'anglais, mais le nombre global de traductions littéraires était faible, avec à peine 300 œuvres traduites dans les deux sens depuis 1580 . Et Stratford de souligner dans son «Introduction» à la réédition que s'il existait une prébistoire de la traduction littéraire au Canada, en revanche, son bistoire n'avait commencé qu'en 1972 avec la création, trois ans après l'adoption de la Loi sur les langues officielles et sous l'égide du Conseil des arts du Canada, du programme d'aide à la

4. N'oublions pas que pouvoir et savoir sont indissociables. Il faut rester vigilant lorsqu'il est question de la volonté des traducteurs anglophones de «faire connaître » la société et la culture québécoises. 
traduction, dont le mandat initial était de multiplier par quatre le nombre de traductions d'œuvres littéraires canadiennes pour atteindre un total de 40 par an. Quant à l'existence d'une tradition de traduction, définie par Stratford comme la traduction systématique d'œuvres littéraires dans les deux langues officielles appuyée par une infrastructure éditoriale et subventionnaire adéquate, elle faisait, selon lui, entièrement défaut. Notons, quitte à y revenir, le recours au singulier : une histoire, une tradition, une infrastructure.

Or, en constatant la non-existence d'une tradition de traduction, Stratford a transformé, chiffres toujours à l'appui, des attitudes sur la traduction littéraire en évidences historiques empiriquement vérifiables. Du coup, deux évolutions possibles se profilaient, «appelées» si l'on peut dire par son constat : l'histoire ultérieure de la traduction littéraire au Canada pouvait évoluer ou bien vers le maintien du statu quo ou bien vers l'émergence d'une véritable tradition de traduction. Selon plusieurs traductologues canadiens-anglais (v. Koustas, 2002; Mezei, 2004), c'est cette deuxième tendance qui a commencé à se dessiner vers le milieu des années 1980, malgré le fait qu'aucune mise à jour de la Bibliographie susceptible de l'entériner n'avait été effectuée ${ }^{5}$ et que les rares statistiques encore fournies (v. Koustas, 1997) avaient fini par être remplacées par des listes plus ou moins complètes censées en tenir lieu : liste des maisons d'édition anglophones et francophones ayant publié des traductions; liste des nouvelles traductions littéraires récemment parues; liste des initiatives lancées depuis la création du programme d'aide à la traduction.

Sans remettre en cause l'importance de tels travaux, soulignons que la conclusion selon laquelle une tradition de traduction littéraire fondée sur la reconnaissance et l'échange mutuels avait été établie (Koustas, 2002, p. 1129) relève du postulat, dont la valeur de vérité reste toujours à démontrer. Soulignons également que, sauf erreur, aucune définition claire de ce qu'il convenait d'entendre par tradition de traduction n'a été proposée, à l'exception de celle de Stratford. Tout au plus et à l'instar des listes sur lesquelles elle s'appuyait, cette expression reconduisait l'impératif d'unité nationale avec les valeurs libérales de délibération consensuelle et de cohabitation pacifique qui l'informent, notamment le principe de l'égalité des deux langues officielles dont les assises juridiques et législatives se trouvent dans la Loi sur les langues officielles (1969), la Charte des droits et des libertés (1982) et la Loi sur le multiculturalisme canadien (1988).

5. C'est toujours le cas en 2018. 
Car c'est bien d'une vitalité interculturelle nationale et officielle qu'il s'agit, de l'image d'un pays officiellement bilingue (se) traduisant systématiquement du français vers l'anglais et de l'anglais vers le français en fonction d'un contrat social partagé qui efface les rapports de force entre langues majoritaire et minoritaire ${ }^{6}$. Affirmer l'existence d'une tradition de traduction permet de construire une histoire (idéalisée) de la traduction littéraire au Canada depuis 1972 qui va de l'antagonisme à l'entente paritaire, de l'indifférence à la reconnaissance mutuelle, de la «nontraduction» conçue comme compromis à la traduction subventionnée donnée pour consensuelle et égalitaire - en un mot, de l'absence d'une tradition de traduction littéraire à une tradition investie par les idéaux politiques inscrits dans la Loi sur les langues officielles à laquelle sont tenues d'adhérer toutes les institutions fédérales.

Fait significatif qui fera l'objet de la suite de cet article : cette vitalité interculturelle nationale se retrouverait à plus petite échelle au Québec, surtout à Montréal où réside $80 \%$ de la minorité anglophone de la province. Comme l'a avancé Sherry Simon (2012, p. 135), «the almost automatic inter-translation of English-language and French-language fiction [...] has become a feature of today's Montréal», faisant de celle-ci une véritable ville traduisante. Si par "quasi-automatique» on peut inférer l'existence d'une tradition de traduction locale, il reste qu'à son tour ce constat, dont la valeur de vérité semble tenue pour acquise, n'est validé par aucune étude empirique. Qui pis est, il refoule dans un angle mort l'histoire des rapports on ne peut plus antagonistes entre les communautés majoritaire et minoritaire du Québec, la rapide dévitalisation culturelle des communautés anglophones entre 1976 et 1995, suivie de leur revitalisation graduelle. Que la traduction littéraire ait contribué à leur revitalisation semble également tenu pour acquis, cette première étant présentée comme vecteur de parité et d'échanges consensuels entre les deux communautés linguistiques. Voici précisément ce que la bibliométrie permet de problématiser en exposant le contenu refoulé de cet angle mort, soit un ensemble de faits empiriques qui à la fois rétablissent l'historicité de tels constats et relativisent la valeur de vérité acquise qu'ils véhiculent.

6. Cette image offre un bon exemple d'adéquation (imaginaire) entre régime linguistique (bilinguisme officiel) et régime traductif (égalité de statut des langues de traduction). Rappelons que le Québec n'a toujours pas consenti à l'adoption de la loi constitutionnelle de 1982, d'où l'hypothèse que la notion d'une tradition de traduction serait une construction de la majorité anglophone. 


\section{Hypothèses, questions de recherche, établissement du corpus, mise en contexte}

Reprenons : le gommage des rapports de pouvoir effectué par la notion de tradition de traduction conduit au postulat de la vitalité mutuelle des deux communautés «fondatrices» sur le double plan national et local. L'idée même de minorité culturelle (francophone au niveau national; anglophone au Québec ${ }^{7}$ ), ainsi que les revendications culturelles que le statut de minorité rend possibles sont reléguées en deçà du seuil consensuel établi par le discours dominant. ${ }^{8}$ Bien que l'affirmation (implicite ou explicite) de l'existence d'une tradition de traduction ne soit pas a priori fausse, on est néanmoins en droit de se demander jusqu'à quel point - et de quel point de vue - elle est vraie. Sur quels critères cette affirmation se fonde-t-elle?

Première hypothèse : cette affirmation est un construit discursif qui repose sur des perceptions, croyances et représentations qui se donnent pour autant d'évidences d'une vitalité interculturelle redevable moins d'un état de fait que de l'idéologie du bilinguisme officiel et des droits individuels. Sans nier que, dans le contexte canadien, la traduction littéraire puisse servir d'indice de collaboration et de vitalité interculturelle ${ }^{9}$, répétons que l'absence de données empiriques a permis d'ériger l'indice en preuve. Dès lors, la tâche de l'historien consiste à restituer à la traduction littéraire la fonction de mesure empirique que Stratford lui avait attribuée ${ }^{10}$.

Deuxième hypothèse : si toute traduction fournit un ensemble d'éléments quantifiables que l'on peut chercher à interpréter, inversement, la valeur de vérité des perceptions interculturelles peut se mesurer à l'aune d'éléments quantifiables. D'où un rapport dialectique entre faits empiriques et idées reçues sur la traduction littéraire qu'il convient d'interroger dans une perspective historique, en admettant

7. Les anglophones représentent environ $13 \%$ de la population du Québec, tandis que celle-ci représente environ $25 \%$ de la population du Canada.

8. On pourrait s'étonner d'un tel gommage, la Loi sur les langues officielles stipulant que «le gouvernement fédéral s'engage à favoriser l'épanouissement des minorités francophones et anglophones du Canada et à appuyer leur développement, ainsi qu'à promouvoir la pleine reconnaissance et l'usage du français et de l'anglais dans la société canadienne» (Partie IV, art. 41, sec.1).

9. Nous distinguerons désormais entre la collaboration interculturelle fondée sur la parité et la collaboration (inter)culturelle fondée sur la prise en compte de rapports de force non paritaires.

10. De tels travaux ont déjà été entrepris dans le contexte canadien à partir de corpus partiels (v. Courcy, 2007; Koustas, 2008). 
avec Lieven D'hulst (2014, p. 99) que l'analyse institutionnelle mérite d'être corrélée à l'analyse discursive, d'une part, et, de l'autre, que l'historicité de la traduction concerne aussi bien le temps de la production que le temps de la réception.

La bibliométrie fournit, nous l'avons dit, un outil heuristique prometteur, à la condition d'avoir accès aux données auxquelles l'appliquer. Or, tel ne fut pas le cas lorsque nous avons voulu y recourir dans le cadre d'un projet de recherche sur la traduction française d'œuvres de fiction pour adultes ${ }^{11}$ publiées par des écrivains de la minorité anglophone du Québec depuis 1976 (v. infra); projet dont l'objectif était de relancer, à ce niveau très local, les travaux de Stratford en vue de vérifier nos hypothèses de départ. Il manquait à la fois une bibliographie complète des œuvres de fiction angloquébécoises publiées depuis cette date et une bibliographie complète de leurs traductions françaises. La première étape du projet a donc consisté à établir un répertoire bibliographique aussi exhaustif que possible (v. Lane-Mercier, 2014b), susceptible de renseigner sur 1) l'ensemble des conditions démographiques, sociales, historiques, économiques et politiques de possibilité de la traduction de la fiction anglo-québécoise depuis 1976 (v. Sapiro, 2008; D’hulst, 2014); 2) les rapports de domination qui les informent (p. ex., délais de traduction; maisons d'édition subventionnées ou non; étendue plus ou moins restreinte des aires de distribution; incidence des prix littéraires); 3) le taux d'échanges et les effets que ces derniers exercent à court, moyen et long terme sur le «degré de pénétration [d'une culture] en une autre» (D'hulst 2014, p. 125).

Comprenant 27 variables, soit 16 pour les œuvres originales et 11 pour les traductions lorsquelles existent, la bibliographie une fois constituée a permis, lors de la deuxième étape, de procéder à une série d'analyses "comptables» en vue de répondre à des questions empiriques précises telles : qui a publié quoi? Quand? Où? Qui a été traduit? Par qui? Quand? Où? Dans quels délais? Qui a reçu une subvention à la traduction? Un prix? Quelles œuvres ont été réimprimées? Retraduites? Quels auteurs ont été traduits une (deux, etc.) fois? Qui n'a jamais été traduit? Ensuite, une série d'analyses statistiques a permis d'identifier, en fonction d'une ou plusieurs variables, des probabilités (p. ex. la probabilité de traduction si l'original a été primé) et les tendances significatives.

11. "Euvres de fiction" désigne ici le roman, le récit et le recueil de nouvelles. Ont été écartés la littérature de jeunesse, le roman graphique, la bande dessinée, tout ouvrage de moins de 50 pages et les œuvres numériques sans édition papier. 
La troisième étape, toujours en cours, consiste à mener des analyses qualitatives en recourant aux outils méthodologiques et conceptuels de la traductologie. Quelles sont les tendances qui s'y profilent? Comment les interpréter? Quelles attitudes, croyances ou évidences sur la traduction sont confirmées, remises en cause, contredites ou encore engendrées par l'approche bibliométrique elle-même, selon l'hypothèse de la dialectique du fait empirique et de l'idée reçue évoquée plus haut? Autrement dit, qu'est-ce que la bibliographie permet de dire sur le rôle de la traduction littéraire dans la revitalisation culturelle des communautés anglophones du Québec, sur les processus de (non-)transfert, de (non-)réception ou de (non-) consécration de leur littérature, sur les rapports (inter)culturels entre communautés majoritaires et minoritaires aux niveaux local (Québec), national (Canada), voire international ?12 En admettant que l'approche bibliométrique dévoile la persistance de rapports de domination là où «on» ne les voyait plus, est-il possible d'envisager une relecture de l'histoire de la traduction littéraire au Canada, laquelle se doublerait d'une relecture de l'histoire des rapports (inter) culturels entre ses deux communautés linguistiques officielles? Sans pouvoir répondre ici à toutes ces questions, nous présenterons un aperçu de quelques tendances qui révèlent les déséquilibres que la notion de tradition de traduction nivelle afin de se conformer à l'idéologie pancanadienne dominante. Mais auparavant, une mise en contexte de la littérature anglo-québécoise s'impose.

Consécration du mouvement séparatiste par la majorité francophone, la victoire du Parti Québécois aux élections provinciales de 1976 a provoqué un sentiment de double isolement chez les écrivains anglophones qui jusque-là avaient fait partie intégrante de l'élite économique du Québec et de l'establishment littéraire canadienanglais sans trop se soucier de leurs rapports aux milieux culturels francophones. D'un côté, ils ont compris que, pour les Canadiens anglais, “'English-Canadian' seems no longer to include anglophone Quebeckers» (Davey, 1997, n. p.); de l'autre côté, l'adoption par le gouvernement québécois de la Loi sur la langue officielle (1974) et de la Charte de la langue française (1977) qui, respectivement, fait du français la seule langue officielle de la province et limite les droits des anglophones, leur a conféré le statut de minorité linguistique. Dans ce nouveau contexte, ils se sont sentis marginalisés par rapport

12. Pour une analyse préliminaire des effets sur le plan international, v. LaneMercier, 2018a. 
à l'institution littéraire québécoise majoritaire, à plus forte raison que le concept de québécité, réservé aux francophones, excluait toute autre langue et communauté culturelle. Rares sont les anglophones qui défendront la légitimité de la Charte, faisant simultanément prévaloir leurs droits individuels et leurs droits collectifs acquis (v. Lubin, 2011), ce qui n'a pas empêché la dévitalisation rapide de leurs communautés culturelles au cours des vingt prochaines années.

Ce n'est qu'à partir du milieu des années 1990 que ces communautés ont commencé à se doter d'une infrastructure institutionnelle en vue de promouvoir les œuvres de leurs écrivains tant au Québec qu'au Canada anglais, ce qui a eu pour effet supplémentaire de leur assurer une plus grande présence dans les milieux culturels francophones, en partie grâce à la traduction. Aussi peut-on constater depuis 2000 une ouverture des institutions culturelles québécoises, dont certaines tendent à considérer que les œuvres anglo-québécoises, traduites en français ou non, font bel et bien partie de la littérature québécoise ${ }^{13}$; ouverture qui s'accompagne d'une décrispation significative des rapports (inter)culturels de part et d'autre : non seulement l'ère de la «nontraduction" contestataire semble révolue, mais de nombreux écrivains anglo-québécois estiment désormais avoir plus d'affinités culturelles avec le Québec qu'avec le Canada anglais.

Partie intégrante du processus de revitalisation culturelle, cette décrispation institutionnelle est corroborée par un rapport préparé en 2012 pour Industrie Canada, qui définit l'économie créative comme un processus communautaire mesurable composé d'activités allant de la création à la consommation en passant par la production, la distribution et la vente. Si celles-ci stimulent la croissance économique, elles favorisent aussi l'inclusion sociale et la diversité culturelle :

Les anglophones québécois constituent la plus importante communauté minoritaire de langue officielle provinciale au Canada. Au cours de ces dernières décennies, la composition de cette population est devenue de plus en plus complexe et multiculturelle. Parallèlement à ces changements démographiques, l'influence de la culture anglophone québécoise a aussi évolué [... et] a progressivement été acceptée par le gouvernement québécois et par le public francophone. (Zhang, 2012, p. i, 2)

13. L'idée de l'appropriation de la littérature anglo-québécoise traduite mérite d'être approfondie (v. Leconte, 2017). 
Pour sa part, le directeur exécutif du English-Language Arts Network souligne l'importance à renforcer la vitalité culturelle de la minorité anglo-québécoise en développant

working relationships with our Francophone colleagues in an environment where increased vitality of the English language is often seen as a threat to French [...]. Our priority is to establish the 'brand' of English-language culture as a positive and creative force within Quebec. (Rodgers, cité par Fraser, 2011, n. p.)

On retrouve ici, outre le rôle assuré par des facteurs démographiques, institutionnels et économiques, l'idée de collaborations (inter) culturelles locales fondées sur le compromis, la volonté d'inclusion et la reconnaissance explicite des rapports de force («majorité»; «minorité»; «threat») conçus aussi bien comme garants de la diversité culturelle effective que comme source de compromis "créatifs". L'image de marque («brand») de la culture anglo-québécoise a besoin d'être forgée avec la majorité francophone dans une volonté de participation citoyenne qui se positionne contre la majorité canadienne-anglaise.

Effectivement, l'(auto-)perception selon laquelle les écrivains anglo-québécois demeurent marginalisés au niveau national est tenace. En témoigne, entre autres, l'appel à l'action lancé par l'auteure d'un rapport préparé en 2005 pour la Quebec Writers' Federation (QWF) : dans la mesure où ces écrivains «lack a distinct Canadian profile and [...] solid Canadian exposure» (Soderstrom, 2005, n. p.), il est urgent de développer des stratégies pour rehausser leur visibilité; appel qui semble n'avoir rien perdu de son actualité (v. Lane-Mercier, 2018b). Cette perception d'invisibilité se fonde sur la croyance tout aussi tenace que si les éditeurs canadiens-anglais les ignorent, c'est parce que le Canada anglais est persuadé que les écrivains du Québec écrivent uniquement en français (v. Davey, 1997; Leith, 2010) ${ }^{14}$.

Une analyse bibliométrique axée sur des phénomènes mesurables permet d'interroger le bien-fondé empirique de telles perceptions que contredit, entre autres, le fait que depuis 1995 le nombre de subventions octroyées pour la traduction vers le français dépasse le nombre de celles accordées pour la traduction vers l'anglais (v. Lane-Mercier, 2018a). Le moment est donc venu d'esquisser des

14. Leith (2010, p. 152) fournit quelques exemples. Life of Pi de Yann Martel (2001), qui n'a été retenu pour aucun prix canadien-anglais, a gagné le prix de la QWF, suivi du très prestigieux Man Booker Prize. 
éléments de réponse à quelques-unes des questions formulées plus haut, en gardant présente à l'esprit la prémisse selon laquelle la mise au jour des angles morts du consensuel redonne une visibilité aux communautés culturelles minoritaires, dont la survie dépend - on ne saurait assez le dire en régime de bilinguisme officiel - de mesures de revitalisation tributaires d'une reconnaissance positive des rapports de domination qu'escamote un projet de société homogénéisant basé sur l'égalité des cultures dites fondatrices.

\section{Deux exemples ${ }^{15}$}

Entre 1976 et 2016,1148 16 œuvres de fiction anglo-québécoises ont été publiées, dont $232(20,2 \%)$ ont été traduites en français; parmi celles-ci, 139 (59,7\%) ont été subventionnées par le programme d'aide à la traduction ${ }^{17}$ auquel sont admissibles seules les œuvres traduites par un traducteur canadien et publiées au Canada. Le nombre de traductions a fluctué au cours des années, oscillant entre $10,8 \%$ et $28 \%$ par tranche de cinq ans, sans jamais augmenter de manière significative par rapport au nombre d'œuvres non traduites, lequel a pourtant continué de croître. Bien au contraire, le pourcentage d'œuvres traduites, qui a plafonné entre 1990 et 1995, a commencé à chuter dès le lendemain du deuxième referendum sur la souveraineté du Québec (1995 - remporté de justesse par le «non»); chute graduelle qui, paradoxalement, coïncide avec les débuts de la revitalisation culturelle des communautés anglophones. En effet, celle-ci est attestée sur le plan littéraire par l'augmentation constante du nombre d'originaux publiés depuis le référendum, alors que l'écart entre œuvres traduites et non traduites se creuse progressivement (voir tableau 1, page suivante).

Cet écart peut être attribué à plusieurs facteurs : 1) les originaux publiés en ligne à compte d'auteur, dont le nombre augmente de plus en plus, attirent rarement les éditeurs francophones et ne sont généralement pas recensés; 2) certaines maisons d'édition anglophones ont des réseaux de distribution très réduits et n'attirent

15. Cette section met à jour, en y apportant des précisions supplémentaires, des analyses déjà effectuées (v. Lane-Mercier 2014b, 2018a, 2018b).

16. Tous les chiffres contenus dans cette section ont été compilés en décembre 2016 et doivent être revus à la lumière, notamment, de nouvelles traductions parues depuis cette date.

17. Les traductions subventionnées incluent 8 originaux qui n'ont jamais été publiés. 
pas non plus l'attention des éditeurs francophones; 3) les éditeurs québécois publient également des traductions d'œuvres canadiennesanglaises (v. infra.); 4) jusqu'à récemment, les éditeurs canadiens pouvaient obtenir un maximum de six subventions par année du programme d'aide à la traduction - le nombre est dorénavant de huit -, ce qui impose une limite institutionnelle même si le nombre de traductions non subventionnées reste élevé $\left(40 \%{ }^{18} ; 5\right)$ les critères d'admissibilité aux subventions excluent certaines maisons d'édition; 6) les originaux publiés depuis 2013 sont toujours de bons candidats à la traduction ${ }^{19}$.

\section{Tableau 1. Nombre d'œuvres anglo-québécoises traduites et non traduites, 1976-2016}

\begin{tabular}{|l|l|l|l|l|}
\hline DATES & $\begin{array}{l}\text { Euvres } \\
\text { publiées }\end{array}$ & Traduites & Non traduites & $\%$ Traduites \\
\hline $1976-1980$ & 37 & 4 & 33 & $10,8 \%$ \\
\hline $1981-1985$ & 59 & 12 & 47 & $20,3 \%$ \\
\hline $1986-1990$ & 82 & 17 & 65 & $20,7 \%$ \\
\hline $1991-1995$ & 107 & 30 & 77 & $28 \%$ \\
\hline $1996-2000$ & 125 & 32 & 93 & $25,6 \%$ \\
\hline $2001-2005$ & 164 & 39 & 125 & $23,8 \%$ \\
\hline $2006-2010$ & 242 & 53 & 189 & $22 \%$ \\
\hline $2011-2016^{20}$ & 332 & 45 & 287 & $13,6 \%$ \\
\hline
\end{tabular}

Cela étant, l'écart actuellement spectaculaire depuis 2011 entre le nombre d'œuvres traduites $(13,6 \%)$ et non traduites $(86,4 \%)$ pointe vers des processus de revitalisation et de participation à la société québécoise où la traduction littéraire - pratique interculturelle par excellence - n'a jamais été appelée à jouer un rôle de premier plan. Du coup, la traduction n'est ni indice clair de vitalité culturelle des

18. Ce pourcentage s'explique en grande partie par le fait que les traductions publiées en France, assez nombreuses, ne sont pas admissibles aux subventions du Conseil des arts (v. tableaux 2 et 3 ).

19. Une analyse plus complète des raisons possibles pour cet écart devrait tenir compte des tirages et des ventes, données que nous n'avons pas encore réussi à obtenir.

20. Nous incluons ici les chiffres pour 2016 : 46 œuvres publiées; 4 traduites, soit moins de $10 \%$. 
communautés anglophones, ni preuve de collaborations interculturelles quasi automatiques avec la majorité francophone, encore moins confirmation de l'existence d'une tradition de traduction à l'échelle nationale. En revanche, elle a le mérite d'avoir ouvert une brèche tout à la fois dans le mono-culturalisme du concept de québécité, le monolinguisme de la Charte de la langue française et le principe de l'égalité de l'anglais et du français inhérent au bilinguisme officiel fédéral. De ce point de vue, si le nombre instable de traductions dément l'existence d'une tradition, le fait que 20,2\% des œuvres anglo-québécoises parues depuis 1976 ont tout de même été traduites est symptomatique du rôle positif des déséquilibres (inter) culturels qui, à partir du moment où ils donnent droit de cité à ce qui se situe en deçà du seuil consensuel, se transforment en vecteurs de concessions réciproques susceptibles d'assurer à la culture minoritaire une visibilité et un sentiment d'appartenance essentiels à sa revitalisation. Or, cette interprétation doit être nuancée.

Deuxième exemple : le lieu de publication des originaux a un impact significatif sur la probabilité de traduction; aussi s'avère-t-il une mesure pertinente de vitalité (inter)culturelle :

Tableau 2. Nombre d'œuvres originales selon le lieu de publication, 1976-2016

\begin{tabular}{|l|l|l|l|l|}
\hline DATES & Montréal & Toronto & $\begin{array}{l}\text { Ailleurs } \\
\text { au Canada } \\
\text { anglais }\end{array}$ & $\begin{array}{l}\text { À l'étranger } \\
\text { (New York et } \\
\text { Londres })\end{array}$ \\
\hline $1976-1980$ & 2 & 11 & 17 & 7 \\
\hline $1981-1985$ & 15 & 20 & 10 & 14 \\
\hline $1986-1990$ & 20 & 28 & 25 & 9 \\
\hline $1991-1995$ & 31 & 33 & 28 & 15 \\
\hline $1996-2000$ & 35 & 37 & 34 & 19 \\
\hline $2001-2005$ & 46 & 54 & 36 & 30 \\
\hline $2006-2010$ & 76 & 64 & 47 & 55 \\
\hline $2011-2016$ & 96 & 95 & 57 & 84 \\
\hline TOTAL & $\mathbf{3 2 1}$ & $\mathbf{3 4 2}$ & $\mathbf{2 5 4}$ & $\mathbf{2 3 3}$ \\
& $\mathbf{2 8 \%}$ & $\mathbf{2 9 , 8} \%$ & $\mathbf{2 2 , 1 \%}$ & $\mathbf{2 0 , 3} \%$ \\
\hline
\end{tabular}

21. 41 œuvres ont été publiées à l'extérieur de Montréal (3,6 \%). 
On remarquera que si Toronto mène en termes de chiffres absolus, l'industrie du livre anglophone au Québec, fortement centralisée à Montréal, a connu une croissance marquée depuis 2006, si bien qu'à l'heure actuelle l'écart entre les métropoles s'est refermé. Au Canada anglais, on constate par ailleurs un intérêt grandissant de la part de maisons situées à l'extérieur de Toronto, que ce soit ailleurs en Ontario (115 publications) ou dans d'autres provinces (139 publications). La tendance depuis 2011 est particulièrement frappante: en tenant compte, d'une part, de l'augmentation d'œuvres publiées (tableau 1) et, d'autre part, du nombre non négligeable parmi celles-ci publiées au Canada anglais (596, soit $52 \%$ ), tout porte à croire non seulement que les auteurs anglo-québécois cherchent activement des éditeurs canadiens-anglais, mais qu'il y a un intérêt qui va en augmentant pour la fiction anglo-québécoise à l'échelle nationale. Ces chiffres contredisent, et ce, dès l'arrivée au pouvoir du Parti Québécois en 1976, le sentiment d'isolement et d'invisibilité que bon nombre d'auteurs anglo-québécois continuent à déplorer.

On pourrait néanmoins être tenté de conclure à la réussite des stratégies de branding réclamées par la QWF il y a à peine une dizaine d'années. Or, un éventuel bémol s'élève : jusqu'à quel point les éditeurs canadiens-anglais soulignent-ils à l'intention de leurs lecteurs l'identité de ces écrivains du Québec ou, au contraire, la passent-ils tout simplement sous silence, ce qui viendrait exacerber, en dépit des chiffres, leur sentiment d'invisibilité? Voici un bel exemple de la dialectique du fait empirique et des idées reçues : si ces dernières n'ont pas valeur de vérité absolue, elles ne sont pas totalement non fondées. Quoi qu'il en soit, se profilent ici de nouvelles brèches sous forme de luttes potentielles tant symboliques quéconomiques entre l'institution littéraire anglophone du Québec en forte expansion et l'establishment littéraire canadien-anglais largement associé aux maisons torontoises; luttes qui signalent une fracture interne à «la» littérature canadienne-anglaise que l'intégration à la société québécoise de plus en plus affichée par les écrivains anglo-québécois semble étayer et que le tableau 1 confirme.

Cela dit, le tableau 3 (page suivante) montre qu'une œuvre anglo-québécoise publiée à Toronto - voire au Canada anglais en général - a $40 \%$ de chances d'être traduite en français comparé à Montréal, où la probabilité de traduction est plutôt faible; il en va de même pour les originaux publiés aux États-Unis ou en Angleterre, 
où la probabilité de traduction est de $30 \%$. Ce tableau oblige à relativiser à son tour l'image de Montréal ville traduisante ; image qui, si elle capte la texture des échanges quotidiens et l'impromptu des traversées de l'espace urbain (v. Simon 2012), est dès lors contredite par des processus interculturels institutionnalisés. Résumons : non seulement peu d'œuvres anglo-québécoises sont traduites chaque année à l'échelle du pays, mais celles publiées à Montréal ont peu de chances - moins de $10 \%$ - de lêtre un jour ${ }^{22}$.

\section{Tableau 3. Nombre d'œuvres traduites et non traduites selon le lieu de publication}

\begin{tabular}{|l|l|l|l|l|l|l|l|l|}
\hline DATES & \multicolumn{2}{|l|}{ Montréal $^{23}$} & \multicolumn{2}{l|}{ Toronto } & \multicolumn{2}{l|}{$\begin{array}{l}\text { Ailleurs au } \\
\text { Canada anglais }\end{array}$} & \multicolumn{2}{l|}{ À l'étranger } \\
\hline & trad. & $\begin{array}{l}\text { non } \\
\text { trad. }\end{array}$ & trad. & $\begin{array}{l}\text { non } \\
\text { trad. }\end{array}$ & trad. & $\begin{array}{l}\text { non } \\
\text { trad. }\end{array}$ & trad. & $\begin{array}{l}\text { non } \\
\text { trad. }\end{array}$ \\
\hline $1976-1980$ & 0 & 1 & 2 & 9 & 0 & 17 & 2 & 5 \\
\hline $1981-1985$ & 0 & 12 & 6 & 14 & 2 & 8 & 4 & 10 \\
\hline $1986-1990$ & 2 & 16 & 10 & 18 & 3 & 22 & 3 & 6 \\
\hline $1991-1995$ & 7 & 21 & 16 & 17 & 3 & 25 & 3 & 12 \\
\hline $1996-2000$ & 3 & 20 & 12 & 25 & 10 & 24 & 7 & 12 \\
\hline $2001-2005$ & 5 & 36 & 17 & 37 & 3 & 33 & 14 & 16 \\
\hline 2006-2010 & 4 & 69 & 28 & 36 & 5 & 42 & 16 & 39 \\
\hline 2011-2016 & 4 & 82 & 22 & 73 & 1 & 46 & 17 & 67 \\
\hline TOTAL & $\mathbf{2 5}$ & $\mathbf{2 9 6}$ & $\begin{array}{l}\mathbf{1 1 3} \\
\mathbf{8 , 4} \%\end{array}$ & $\mathbf{2 2 9}$ & $\mathbf{2 7}$ & $\mathbf{2 1 7}$ & $\mathbf{6 6}$ & $\mathbf{1 6 7}$ \\
\hline
\end{tabular}

Il convient par conséquent de reposer la question de savoir jusqu'à quel point les collaborations (inter)culturelles entre anglophones et francophones du Québec "passent» par la traduction lit-

22. Si l'on tient compte du fait que la majorité des traducteurs littéraires canadiens - tant anglophones que francophones - habitent à Montréal, ce dernier chiffre pourrait étonner. Se posent alors la question de la qualité et de l'intérêt (esthétique ou autre) des originaux, ainsi que celle de la taille des maisons d'édition anglo-montréalaises, plus petites et perçues comme moins prestigieuses que les maisons torontoises (v. infra). Aussi des analyses statistiques plus fines sontelles nécessaires, la qualité littéraire et le prestige étant également mesurables. Il faudrait exploiter d'autres variables pour déterminer, par exemple, si les originaux publiés à Toronto ont gagné plus de prix que ceux publiés à Montréal, s'ils se sont vendus à plus d'exemplaires, s'ils ont bénéficié de plus de recensions critiques ou de mentions dans la presse culturelle.

23. Depuis 1976, seulement deux traductions ont paru à l'extérieur de Montréal. 
téraire, d'autant plus que la vigueur actuelle des maisons d'édition anglo-québécoises est consolidée par le fait qu'elles sont désormais en mesure de se positionner, du moins sur le plan quantitatif, par rapport aux prestigieuses maisons torontoises, sans toutefois qu'il y ait des répercussions sur le nombre de traductions vers le français, bien au contraire. La preuve : depuis le début de la revitalisation culturelle (1996-2000), 16 œuvres publiées à Montréal ont été traduites, comparé à 98 parues au Canada anglais, indication du peu de collaborations réelles entre éditeurs anglophones et francophones du Québec, ces derniers s'adressant de prime abord aux éditeurs ontariens lorsqu'ils désirent publier des traductions d'auteurs angloquébécois. Du coup, se dessinent à leur tour d'éventuelles luttes au sein de l'industrie québécoise du livre dans son ensemble, chaque communauté linguistique s'efforçant d'acquérir un maximum de distinction culturelle et, partant, de parts du marché local que la traduction serait susceptible de brouiller. Il est fort possible que ces collaborations limitées servent les intérêts des éditeurs angloquébécois dans la mesure où ceux-ci misent sur le taux élevé de bilinguisme du lectorat francophone pour augmenter leurs ventes (argument commercial) et où ils peuvent ainsi se distancier du phénomène d'appropriation des œuvres traduites déjà évoqué (argument symbolique), a fortiori lorsque les originaux sont «faits au Québec» et qu'il est crucial de revendiquer leur différence, autre synonyme de visibilité et de vitalité renouvelée de la culture minoritaire.

À titre indicatif, la distance prise par rapport à la traduction dans le processus de revitalisation (inter)culturelle est manifeste dans les catalogues de trois maisons d'édition anglo-québécoises qui ont des politiques de publication quasi identiques quant à la place réservée aux auteurs anglophones locaux et à celle réservée aux traductions anglaises d'œuvres québécoises :

Véhicule Press (fondée en 1973) a publié

- 16 œuvres de fiction, dont 8 (50\%) titres angloquébécois

- 3 romans québécois en traduction anglaise

DC Books (fondée en 1992) a publié

- 39 œuvres de fiction, dont 28 (70\%) titres angloquébécois

- 1 roman québécois en traduction anglaise 
Linda Leith Publishing (fondée en 2012) a publié

- 20 œuvres de fiction, dont 14 (70\%) titres angloquébécois

- 2 romans québécois en traduction anglaise

Mais changeons de perspective. Comme nous l'avons suggéré ailleurs (Lane-Mercier, 2018b) et comme le confirment les données présentées au tableau 3, le peu de collaborations (inter)culturelles locales ne signifie pas absence d'intérêt de la part des éditeurs francophones pour la fiction anglo-québécoise, à la condition que celleci arrive au Québec en traduction française via le Canada anglais, en particulier Toronto, y compris lorsque le traducteur habite le Québec. Autrement dit, tout porte à croire que la synergie entre les deux communautés culturelles officielles se déploie avant tout sur le plan national. Plusieurs hypothèses pourraient être avancées, dont surtout celle selon laquelle les écrivains anglo-québécois sont désormais trop intégrés à la société québécoise pour que leurs œuvres affichent le coefficient d'altérité culturelle dont a besoin la traduction, laquelle continue à être conçue en termes de dichotomies paritaires et consensuelles issues de la Loi sur les langues officielles, que le Québec, officiellement monolingue, n'a jamais entérinées. Dans cette optique, le faible intérêt pour la traduction d'originaux publiés à Montréal signifierait l'omniprésence de relations locales davantage transculturelles, définies moins en fonction de dualités qu'en fonction d'allégeances culturelles multiples aptes à relativiser les barrières linguistiques. Si tel est le cas, louverture des institutions culturelles québécoises à l'égard de la littérature anglo-québécoise attestée depuis la fin des années 1990 se serait effectuée, sur le plan local, selon d'autres voies que celle offerte par la traduction littéraire.

Par contraste, l'Anglo-Québécois, explicitement ou implicitement délesté de sa "québécité» une fois publié à Toronto, "assimilé» à la littérature canadienne-anglaise (au singulier), retiendrait une aura de différence qui appelle, de nouveau, la reconnaissance interculturelle et, par-là, les solutions de compromis qu'offre la traduction. Par ailleurs, il ne faut pas écarter - loin s'en faut - l'hypothèse à la fois économique et symbolique selon laquelle les œuvres publiées par les maisons torontoises bénéficient d'une plus-value de distinction relativement à celles publiées par les maisons anglophones du Québec, moins prestigieuses et aux réseaux de distribution moins larges ou robustes. Il se peut cependant que, malgré l'écart toujours 
plus important entre le nombre d'œuvres anglo-québécoises publiées et le nombre de traductions, les données empiriques témoignent aussi de la vitalité de la société québécoise dans son ensemble, à laquelle contribuent, chacune à sa façon, les deux communautés linguistiques dont les intersections culturelles, devenues moins tendues, plus fluides et plus aléatoires, tendent à (ou peuvent se permettre de) se passer de traduction lorsque leurs écrivains publient au Québec.

Si la dialectique des faits empiriques et des représentations discursives est à même de dénoncer les idées reçues sur le rôle assumé par la traduction littéraire dans la revitalisation culturelle des communautés anglo-québécoises et dans l'ouverture des majorités francophone (du Québec) et anglophone (du Canada) à leur égard, cette dialectique produit de nouvelles représentations (inter)culturelles qu'il importe de vérifier à l'aide de chiffres. La variable du lieu de publication des originaux offre par conséquent un excellent exemple de la manière dont une articulation entre l'histoire de la traduction et la traductologie peut s'envisager : aux traductologues de valider les hypothèses que les données empiriques permettent d'échafauder; aux historiens de remettre en question les représentations dont toute communauté culturelle minoritaire a besoin pour revendiquer son (ses) mode(s) d'appartenance à la majorité en tournant à son avantage les rapports de domination qui, ce faisant, lui permettent de s'auto-déterminer.

\section{Conclusion}

Dans un article paru en 2004, la traductologue canadienne Kathy Mezei notait ceci : «At the beginning of this twentieth-first century, how do we stake out a history of literary translation in Canada? [...] Is there a specific Canadian translation experience?» (2004, p. 107) Et Mezei de répondre à l'affirmative à la lumière de l'évolution des pratiques et discours traductifs depuis les années 1960 vers l'émergence d'une image positive, non conflictuelle, consensuelle de la métaphore du pont, dorénavant le symbole du dialogue entre pairs. Symptomatique d'une «culture of collaboration " (ibid., p. 115), le dialogue entre traducteur et auteur s'avère être «a significant and recurring practice in literary translation» (ibid., p. 114), autrement dit une tradition de traduction qui faisait défaut au moment de la parution de la Bibliographie de Stratford et qui viendrait consacrer le passage de la confrontation à l'interrelation et, au Québec, de la «nontraduction» à la traduction. 
Il n'empêche que, pour être concluants, les nombreux exemples de collaboration cités par Mezei, d'où s'effacent une fois de plus les inégalités et autres points de tension, auraient besoin d'être situés par rapport à l'ensemble des textes traduits et non traduits au cours des quarante dernières années, accompagnés des faits - agents et agences - qui en ont assuré la production, la mise en circulation et la réception des originaux comme des traductions. Sans prétendre que l'évolution des rapports de force sur le plan local du Québec soit identique à l'évolution des rapports de force entre le Canada anglais et le Québec (bien au contraire), la dialectique des faits empiriques et des représentations discursives est également présente sur le plan national où l'on observe depuis 1990 une diminution constante du nombre d'œuvres de fiction traduites du français vers l'anglais accompagnée d'une augmentation tout aussi constante dans l'autre sens. La lecture de l'histoire de la traduction littéraire au Canada proposée en 2004 par Mezei, de même que l'idée de tradition de traduction qui la sous-tend, méritaient donc d'être revisitées afin d'éviter les angles morts du consensuel, comme Mezei (2014, p. 3) l'a reconnu une dizaine d'années plus tard : «The law decrees equality of English and French, and seeks to create a level playing field; however the realities of cultural life are saturated with the conflicting forces of inequality and violence, resistance and redress. ${ }^{24}$

Voici, précisément, la voie ouverte en 1975 par la Bibliographie de Stratford, qui laissait présager une relecture autrement « intranquille » de l'histoire de la traduction littéraire au Canada. Quarante ans après sa publication et à la faveur d'un projet bibliographique inspiré du sien, nous avons voulu poser les jalons d'une relecture fondée non plus sur les conditions de possibilité d'émergence d'une tradition de traduction qui, à l'instar des collaborations interculturelles paritaires, n'est rien d'autre qu'une construction discursive d'ordre politique et juridique conforme aux intérêts de la majorité, mais plutôt sur l'analyse des conditions d'impossibilité de son émergence, que ce soit sur le plan local, national ou international.

24. Frank Davey propose une interprétation percutante de l'échec de l'idéal de parité au sein des universités canadiennes-anglaises: "Anglophone-Canada's agenda to conceal or diminish francophone Quebec, or to separate itself from it, has been covert $[\ldots]$. Anglophones have thus been able to enjoy the various idealisms about Quebec which have informed translation projects, joint conferences [...] and associations like ACQL [Association for Canadian and Quebec Literature], at the same time as we have trained hundreds of Canadian literature specialists to be incapable of participating fully in an association like ACQL.» (1997, n. p.) 
Car ce que nous apprend la bibliographie d'œuvres de fiction anglo-québécoises depuis 1976, grâce à la bibliométrie comme méthode et à la dialectique de l'empirique et du discursif comme hypothèse de départ, c'est la persistance de déséquilibres, de tensions et de ce qui semble être un réseau complexe d'amorces de traditions de traduction - au pluriel - qui ne cessent de freiner l'émergence d'une tradition forte et unique fondée sur la traduction systématique dans les deux langues officielles. Ce que nous « raconte» la bibliographie, c'est une autre histoire - voire d'autres histoires au pluriel - des échanges interculturels entre les anglophones et les francophones de Montréal, du Québec et du Canada que l'histoire officielle, encore tributaire de la Loi sur les langages officielles, n'est pas en mesure de capter. $\mathrm{Ce}$ que nous enseigne la bibliographie, c'est que la revitalisation (inter) culturelle des communautés anglo-québécoises est peu redevable à la traduction littéraire; que Montréal n'est pas une ville traduisante au sens institutionnel du terme; que les écrivains anglo-québécois ne sont pas ignorés par Toronto, mais qu'ils ont peut-être besoin de le croire afin de revendiquer leur légitimité et une image de marque spécifique; que l'idée d'altérité a subi, au Québec, des glissements importants au cours des trente dernières années; que la notion d'une tradition de traduction ne cadre ni avec les faits historiques ni avec les faits traductifs; que la bibliométrie fournit une passerelle puissante entre l'histoire de la traduction et la traductologie.

D'où un nouveau constat: il n'existe pas de tradition de traduction au Canada, en dépit du fait qu'il est le deuxième pays au monde, après la France, à traduire le plus vers le français, tous genres confondus $^{25}$. À la place, on trouve ce que nous venons de nommer des amorces de traditions - toujours au pluriel, toujours instables, toujours en deçà du seuil consensuel - dont le rôle, salutaire dans les contextes de bi- ou de plurilinguisme officiel, consiste à révéler les rapports de domination, les tensions et inégalités qui les soustendent, de même que les croyances et (auto-)perceptions, ni vraies, ni fausses, qui en découlent. Car la vitalité (inter)culturelle de l'ensemble des communautés linguistiques officielles et non officielles en situation minoritaire - voire leur survie - en dépend, afin de débloquer le dialogue qui favorise les compromis réciproques et, ce faisant, récuser les assimilations, marginalisations, effacements et oppressions inhérents tant aux colonialismes qu'aux collaborations interculturelles consensuelles.

25. Voir l'Index Translationum (UNESCO, s. d.), dont les informations les plus récentes remontent à 2009 pour le Canada. 


\section{Références}

Baumgarten, Stefan et Edith Gruber (2014). «Phenomenological Asymmetries in Welsh Translation History». The Translator, 20, 1, p. 26-43.

Brault, Jacques (1975). Poèmes des quatre côtés. Saint-Lambert, Éditions du Noroît.

Cardinal, Linda (2001). «Droits, langue et identité. La politique de la reconnaissance à l'épreuve de la judiciarisation». In J. Maclure et A.G. Gagnon, dir. Repères en mutation. Identité et citoyenneté dans le Québec contemporain. Montréal, Éditions Québec Amérique, p. 269-294.

Cardinal, Linda (2011). "Language Planning and Policy-Making in Quebec: The Difficult Road to Linguistic Peace». In S. Gervais, C. Kirkey et J. Rudy, dir. Quebec Questions. Oxford, Oxford University Press, p. 194-201.

Courcy, Nathalie (2007). «La traduction littéraire au Canada entre 1997 et 2001 : un pont (fragile?) entre deux communautés linguistiques et culturelles». In C. Lombez et R. von Kulessa, dir. De la traduction et des transferts culturels. Paris, L'Harmattan, p. 52-61.

Cronin, Michael (2006). Translation and Identity. Londres, Routledge.

Davey, Frank (1997). "“AND Quebec": Canadian Literature and Its Quebec Questions». Canadian Poetry. Disponible à <www.uwo.ca/ english/canadianpoetry/cpjrn/vol40/davey.htm> [consulté le 10 juillet 2018].

D'hulst, Lieven (2014). Essais d'histoire de la traduction. Avatars de Janus. Paris, Classiques Garnier.

Fraser, Graham (2008). «Quebec's English-Speaking Community: Adapting to a New Social Context». In R. Bourhis, dir. The Vitality of the English-Speaking Communities of Quebec: From Community Decline to Revival. Moncton, Canadian Institute for Research on Linguistic Minorities, p. 221-224.

Fraser, Graham (2011). Annual Report 2010-2011. Disponible à <http:// www.ocolclo.gc.ca/en/publications/annual_reports/2010-2011> [consulté le 10 juillet 2018].

Jedwab, Jack (2008). «How Shall We Define Thee? Determining Who is an English-Speaking Quebecer and Assessing its Demographic Vitality». In R. Bourhis, dir. The Vitality of the English-Speaking Communities of Quebec: From Community Decline to Revival. Moncton, Canadian Institute for Research on Linguistic Minorities, p. 1-18.

Koustas, Jane (1997). «Quebec Literature in Translation: Loaded Canons». Québec Studies, 23, p. 43-53.

Koustas, Jane (2002). «Translation». In W. H. New, dir. Encyclopedia of Canadian Literature. Toronto, University of Toronto Press, p. 1123-1128.

Koustas, Jane (2008). Les Belles Étrangères. Canadians in Paris. Ottawa, University of Ottawa Press. 
Lane-Mercier, Gillian (2014a). «Les carences de la traduction littéraire au Canada : des bibliographies et des traditions». Meta, 59, 3, p. 517-536.

Lane-Mercier, Gillian (2014b). «La fiction anglo-québécoise en traduction française depuis 1990 : agents, agences et textes ». Recherches sociographiques, LV, 3, p. 531-558.

Lane-Mercier, Gillian (2017). "The Role of Institutional Collaborations in Contexts of Official Bilingualism: The Canadian Example." In A. Cordingley et C. Frigau Manning, dir. Collaborative Translation. London, Bloomsbury Publishing, p. 214-234.

Lane-Mercier, Gillian (2018a). «Official Facts and Fictions: The Canada Council's Discourse on Literary Translation (1972-2015)». In J. Woodsworth, dir. The Fictions of Translation. Amsterdam/Philadelphie, John Benjamins, p. 273-296.

Lane-Mercier, Gillian (2018b). «From English into French: Literary Translation as a Measure of the (Inter)Cultural Vitality of Québec's Anglophone Communities». In S. Gervais, R. Iacovino et M. A. Poutanen, dir. Engaging with Diversity: Multidisciplinary Reflections on Plurality from Québec. Berne, Peter Lang, p. 457-476.

Leconte, Marie (2017). «Accéder au champ de la littérature québécoise par la traduction : argumentation, suivie d'un exemple». Québec Studies, 64, p. 3-23.

Leith, Linda (2010). Writing in the Time of Nationalism. From Two Solitudes to Blue Metropolis. Winnipeg, Signature Editions.

Lubin, Martin (2011). «The Politics of Language in Quebec».In S. Gervais, C. Kirkey et J. Rudy, dir. Quebec Questions. Oxford, Oxford University Press, p. 168-183.

Maclure, Jocelyn (2001). «Pluralisme et démocratie : dialogue, décision et dissensus». In J. Maclure et A.-G. Gagnon, dir. Repères en mutation. Identité et citoyenneté dans le Québec contemporain. Montréal, Éditions Québec Amérique, p. 251-267.

Meylaerts, Reine (2009). «"Et pour les Flamands, la même chose" : quelle politique de traduction pour quelles minorités linguistiques?». Meta, 54, 1, p. 7-21.

Meylaerts, Reine (2011). «Translational Justice in a Multilingual World: An Overview of Translational Regimes». Meta, 56, 4, p. 743-757.

Mezei, Kathy (2004). «Dialogue and Contemporary Literary Translation». In D. Saint-Jacques, dir. Tendances actuelles en histoire littéraire canadienne. Québec, Éditions Nota Bene, p. 107-129.

Mezei, Kathy, Sherry Simon et Luise von Flotow (2014). Translation Effects. The Shaping of Modern Canadian Culture. Montréal et Kingston, McGill-Queen's University Press.

Sapiro, Gisèle (2008). Translatio. Le marché de la traduction en France à l'heure de la mondialisation. Paris, Éditions du CNRS. 
Simon, Sherry (1994). Le trafic des langues. Traduction et culture dans la littéraire québécoise. Montréal, Boréal.

Simon, Sherry (2012). Cities in Translation: Intersections of Language and Memory. Londres, Routledge.

Soderstrom, Mary (2005). Quebec English-Language Writers and Publishers Research Project: Final Report. Quebec Writers Federation and The Association of English-Language Publishers of Quebec, August 22, n. p.

Stratford, Philip et Maureen Newman (1977 [1975]). Bibliography of Canadian Books in Translation: French to English and English to French/ Bibliographie de livres canadiens traduits de l'anglais au français et du français à l'anglais. $2^{\mathrm{e}}$ édition, Ottawa, CCRH.

UNESCO (s. d.). Index Translationum. Disponible à <http://www.unesco. org/xtrans/bsstatlist.aspx?m=14> [consulté le 12 juillet 2018].

Vázquez, Rolando (2011). «Translation as Erasure: Thoughts on Modernity's Epistemic Violence». Journal of Historical Sociology, 24, 1, p. 2744.

Weinstock, Daniel (2001). «Les "identités" sont-elles dangereuses pour la démocratie ?» In J. Maclure et A.-G. Gagnon, dir. Repères en mutation. Identité et citoyenneté dans le Québec contemporain. Montréal, Éditions Québec Amérique, p. 227-250.

Zhang, Tracy (2012). L'économie créative et les communautés anglophones au Québec. Montréal, Université Concordia, p.i-54. Disponible à $<$ https://www.ic.gc.ca/eic/site/com-com.nsf/vwapj/economie_cretive_ communautes_anglophones_Quebec.pdf/\$file/economie_cretive communautes_anglophones_Quebec.pdf> [consulté le 10 juillet 2018].

Gillian Lane-Mercier

Département de littératures de langue française, de traduction et de création Université McGill Montréal, Québec, Canada gillian.lane-mercier@mcgill.ca 